\title{
Structural Studies of Trimeric SIV and HIV-1 Envelope Glycoproteins Using Cryo-Electron Tomography
}

\author{
T.A. White, A. Bartesaghi, M.J. Borgnia, M.J.V. de la Cruz, J.L.S. Milne, S. Subramaniam \\ Laboratory of Cell Biology, National Cancer Institute, 50 South Dr., Bldg 50/Room 4306, \\ Bethesda, Maryland 20892
}

About 2.5 million individuals are newly infected with HIV each year, and over 2 million deaths result annually from HIV/AIDS infection (http://www.unaids.org), but the development of an effective vaccine remains elusive. Human and simian immunodeficiency viruses (HIV and SIV, respectively) bind target cells by interaction of the envelope glycoproteins (Env) on the virus with the cell surface receptors, CD4 and CCR5(1). The conformational changes in trimeric Env that are triggered by this interaction lead ultimately to fusion of the viral and cell membranes and delivery of the viral core into infected cells.

Recent advances in cryo-electron tomography to obtain 3D density maps from pleiomorphic biological structures provide new methods to tackle the challenge of describing the structure of trimeric Env as displayed on infectious viruses under near-native conditions (Figure 1). Starting from a series of tilted projection images of plunge-frozen viruses, tomograms that capture the distribution of density on the surface and interior of the virus can be determined. In general, the resolution that can be obtained in a tomogram of a single virus is barely enough to discern molecular shapes because images are recorded at the lowest possible electron doses in order to minimize damage from electron irradiation of the sample (Figure 2). However, by extracting sub-volumes corresponding to each trimeric spike, and accounting properly for the missing wedge of data that is inherent to electron tomography, 3D classification and averaging can be used to obtain density maps at signal-to-noise ratios that are sufficiently high for molecular interpretation. Here, we have used cryo-electron tomography combined with wedge-corrected 3D averaging and reference-free image classification methods $(2)$ to compare structures, at $\sim 20 \AA$ resolution, of trimeric Env present on SIVmneE11S and SIVmac239 viruses. Our new results extend earlier studies of HIV-1 BaL from our laboratory(3), and resolve previous disagreements in the structures of SIV envelope glycoprotein spikes, in addition to providing a map of both trimeric Env complexes at higher resolution.

\section{References}

1. A. G. Dalgleish et al., Nature 312, 763 (Dec 20-1985 Jan 2, 1984).

2. A. Bartesaghi et al., J Struct Biol 162, 436 (Jun, 2008).

3. J. Liu, A. Bartesaghi, M. J. Borgnia, G. Sapiro, S. Subramaniam, Nature 455, 109 (2008).

4. $\quad$ P. Zhu et al., Nature 441, 847 (2006).

5. G. Zanetti, J. A. G. Briggs, K. Grunewald, Q. J. Sattentau, S. D. Fuller, PLoS Pathogens 2, 0790 (2006).

6. P. Zhu, H. Winkler, E. Chertova, K. A. Taylor, K. H. Roux, PLoS Pathogens 4, (2008). 

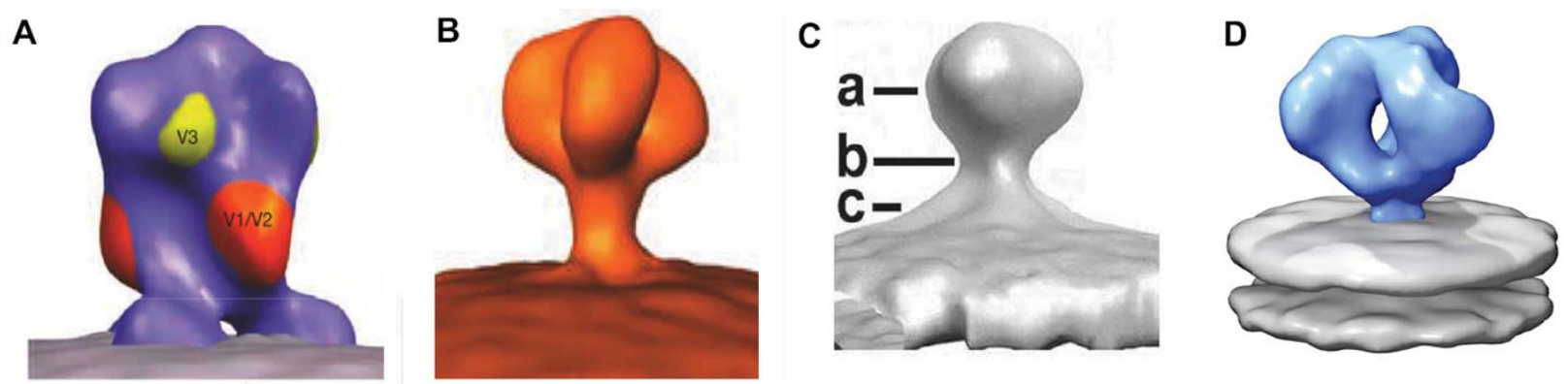

Figure 1. Comparison of previously reported density maps for trimeric Env (A-C) with the map for HIV-1 BaL reported from our laboratory (D) that was obtained using cryo-electron tomography combined with 3D image analysis(3). The previously reported maps are from A) SIV mac239 (Zhu et al(4)), B) SIVmne(Zanetti et al(5)) and C) HIV-1 BaL(Zhu et al(6)).
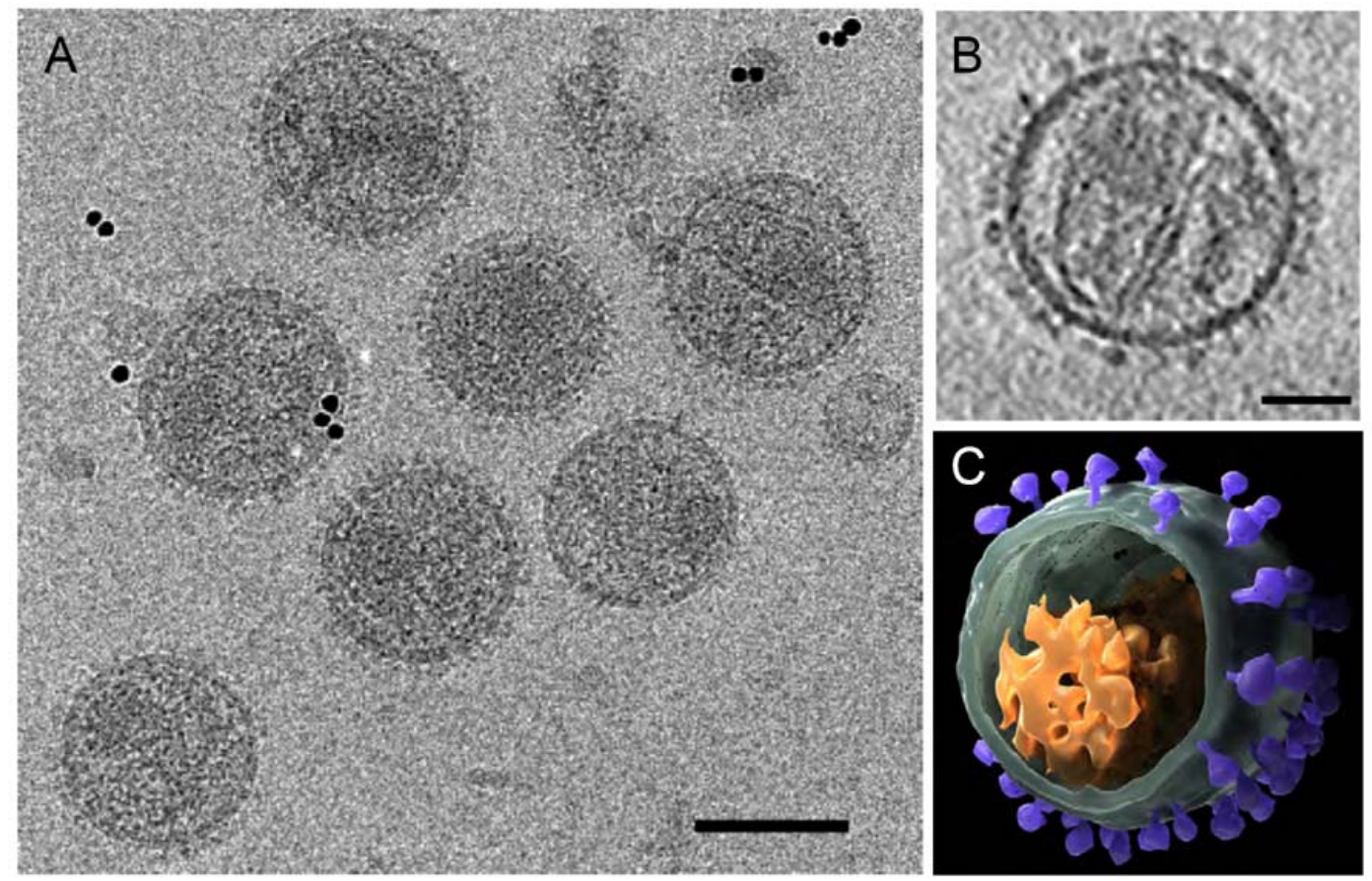

Figure 2. Cryo-electron tomography of SIV A) Low dose $\left(12\right.$ electron $\left./ \AA^{2}\right) 2 \mathrm{D}$ projection image recorded from purified SIVmne virions plunge-frozen in physiological buffer. Gold fiducials $(10 \mathrm{~nm})$ are seen as opaque, round spheres and scale bar is $80 \mathrm{~nm}$. B) Denoised slice $(10 \mathrm{~nm})$ through a reconstructed cryo-electron tomogram (binned $4 \times 4$ ) of a single virus. Scale bar represents $40 \mathrm{~nm}$. C) 3D rendering of virus shown in B) segmented to highlight the viral membrane (green), core (orange) and Env (purple). 